\title{
Avaliação da adesão aos anti-retrovirais em um município no Sul do Brasil
}

\author{
Assessment of adherence to antiretroviral drugs in \\ a municipality in southern Brazil
}

\author{
Carine Raquel Blatt ${ }^{1,3}$, Cecília Bathke Citadin², Fernanda Gaspar de Souza² \\ Rogério Sobrosa de Mello ${ }^{1}$ e Dayani Galato ${ }^{1,3}$
}

\begin{abstract}
RESUMO
0 objetivo desta pesquisa foi avaliar os fatores preditores e o grau de adesão aos anti-retrovirais por meio de auto-relato e data de retirada da medicação. Trata-se de um estudo transversal em que foram entrevistados 67 pacientes. Foram considerados aderentes aqueles pacientes que utilizaram mais de $90 \%$ das doses. Os resultados de adesão foram: referida (72,7\%); calculada por dose esquecida no último dia (70\%); por três dias $(76,1 \%)$; por sete dias $(80,5 \%)$; e por quinze dias $(80,5 \%)$; calculada pela data de retirada da medicação no período de três meses $(53,7 \%)$; $\mathrm{e}$ em seis meses (47,8\%). As variáveis associadas significativamente com a adesão foram: escolaridade, fato de viver com a família, referir boa adesão, avaliação positiva da terapia anti-retroviral, diagnóstico por doença oportunista, menor contagem de linfócitos CD4 maior que 200 células/mm³ e 0 fato de estar no primeiro tratamento. A partir da identificação dos fatores que comprometem a adesão, devem ser elaboradas estratégias individuais e coletivas para a promoção da adesão.
\end{abstract}

Palavras-chaves: Adesão. Terapia anti-retroviral. Vírus da imunodeficiência humana. Síndrome da imunodeficiência adquirida.

\begin{abstract}
The aim of this research is to assess predicting factors and adherence levels to antiretrovirals through self-report and the date of drug retrieval. It is a transversal study in which 67 patients were interviewed. Patients who used more than $90 \%$ of doses were considered to have complied with the treatment. Results of adherence were: self-reports (72.7\%); calculated using dosage forgotten on the last day (70\%); in three (76.1\%) days; in seven $(80.5 \%)$ days; and in fifteen (80.5\%) days; calculated using the date of drug retrieval in a period of three (53.7\%) months; and in six (47.8\%) months. Variables significantly associated with adherence were: educational level, living with the family, refer good adherence, positive assessment of the antiretroviral therapy, a diagnosis of an opportunistic disease, NADIR greater than 200 cells $/ \mathrm{mm}^{3}$ and being in first-time treatment. To improve adherence rates individual and collective strategies should be elaborated taking into account factors which have been identified as negatively effecting adherence.
\end{abstract}

Key-words: Adherence. Antiretroviral therapy. Human immunodeficiency virus. Acquired immunodeficiency syndrome.

No Brasil, a AIDS vem adquirindo proporções cada vez maiores. Entre 1980 e junho de 2007 foram notificados 474.273 casos da doença. Nas Regiões Sul, Sudeste e Centro Oeste, a incidência da síndrome da imunodeficiência adquirida (AIDS) tende à estabilização, no entanto, no Norte e no Nordeste, a tendência é de crescimento ${ }^{19}$. Segundo a Organização Mundial de Saúde (OMS), o Brasil tem uma epidemia concentrada, com taxa de prevalência da infecção pelo vírus da imunodeficiência humana (HIV) de 0,6\% na população de 15 a 49 anos ${ }^{19}$. Portanto, apesar

\footnotetext{
1. Curso de Farmácia e Medicina, Universidade do Sul de Santa Catarina (UNISUL), Tubarão, SC. 2. Curso de Farmácia da Universidade do Sul de Santa Catarina (UNISUL), Tubarão, SC. 3. Núcleo de Pesquisa em Atenção Farmacêutica e Estudos de Utilização de Medicamentos (NAFEUM/UNISUL), Tubarão, SC.

Endereço para correspondência: Profł- Dra. Dayani Galato. Curso de Farmácia UNISUL. Av. José Acácio Moreira 787, Bairro Dehon , 88704-900 Tubarão, SC.

Telefax: 5548 3621-3284

e-mail: dayani.galato@unisul.br

Recebido para publicação em 29/09/2008

Aceito em 20/03/2009
}

dos esforços de prevenção da infecção pelo HIV, a epidemia do vírus continua em expansão ${ }^{16}$.

Embora, em números absolutos, o Brasil tenha registrado 192.709 óbitos por AIDS de 1980 a 2006, dados atuais demonstram que o número de pessoas que morrem com AIDS está diminuindo. Em 2005, o coeficiente de mortalidade por AIDS foi de 6,0/100.000 habitantes para o Brasil, enquanto em 1996 este coeficiente era de 9,6/100.000 habitantes ${ }^{19}$. Isso, em parte, pode ser reflexo da implementação da política de acesso universal e gratuito à terapia anti-retroviral (TARV), implantada desde 1996. o número de pacientes que utilizam anti-retrovirais no Brasil aumentou de 36.000 em 1997 para $170.000 \mathrm{em} 2005^{16}$. 0 tratamento possibilita tanto o aumento da qualidade de vida dos portadores de HIV quanto a diminuição das internações hospitalares decorrentes de infecções oportunistas e, conseqüentemente, a diminuição da mortalidade por AIDS ${ }^{420}$.

Nesse contexto, o maior desafio atual tem sido a adesão ao tratamento anti-retroviral, conceituada como o efetivo uso da medicação segundo o prescritor $^{21}$. 
A medicação tomada de maneira inadequada ou insuficiente (lapsos, falhas nos dias ou nos horários) contribui para a emergência da resistência à terapia farmacológica e para 0 comprometimento dos resultados clínicos ${ }^{7122}$. Para a OMS, as taxas de ingestão devem ser de no mínimo 95\% do total de medicamentos prescritos para a obtenção da efetividade terapêutica ${ }^{21}$. Entretanto, os dados de adesão terapêutica dos doentes de AIDS apresentados na literatura informam que apenas de $37 \%$ a $85,8 \%$ dos pacientes são aderentes ${ }^{321}$.

A partir desses dados alarmantes, muitas estratégias têm sido desenvolvidas para avaliar a adesão terapêutica, entre elas a adoção de medidas laboratoriais para dosagem dos medicamentos no organismo, embalagens com dispositivos especiais que regulem o horário de abertura dos frascos e até a aplicação de questionários que visem à determinação da adesão referida. A análise dessas estratégias tem mostrado que a aplicação de questionários (método indireto) é tão efetiva quanto a adoção de outras técnicas mais onerosas (método direto) ${ }^{61621}$.

Vários fatores são descritos na literatura como predisponentes à não-adesão terapêutica, como os fatores relacionados ao regime terapêutico, ao paciente e ao sistema de saúde $\mathrm{f}^{613142122}$, sendo destacados principalmente o grande número de medicações envolvidas, a complexidade terapêutica do regime de doses e a baixa tolerabilidade dos doentes à medicação anti-retroviral ${ }^{16}$.

Como os fenômenos relacionados à adesão terapêutica são bastante complexos, a definição de estratégias que visem à melhora da adesão terapêutica deve ser realizada com base no perfil dos pacientes atendidos pelo serviço de acompanhamento farmacoterapêutico.

Este trabalho adquire importância quando se predispõe a conhecer a realidade da adesão dos pacientes atendidos em um centro especializado em saúde no Sul do Brasil, região em que trabalhos dessa natureza são inéditos.

Assim, o objetivo desta pesquisa é avaliar os fatores preditores e o grau de adesão ao tratamento com anti-retrovirais por meio de auto-informe e da data de retirada da medicação de pacientes atendidos em uma Unidade de Saúde do sul de Santa Catarina.

\section{MATERIAL E MÉTODOS}

A pesquisa foi realizada no Centro de Atendimento Especializado na Saúde (CAES), localizado no município de Tubarão, na região sul do Estado de Santa Catarina. Esse ambulatório atende pacientes infectados pelo vírus HIV desse município e da região vizinha, que abrange uma população em torno de cento e cinqüenta mil pessoas. No ano de 2006, havia 716 pacientes cadastrados com HIV/AIDS, destes, 250 estavam em tratamento.

0 atendimento aos pacientes é realizado por uma equipe multiprofissional composta de um médico infectologista, uma enfermeira, uma psicóloga, uma farmacêutica, duas assistentes sociais e duas técnicas de enfermagem.

Para a determinação da adesão terapêutica, foi realizado um estudo transversal. 0 cálculo do tamanho da amostra foi realizado com a fórmula descrita por Arcos Cebrián e Del Mar
Rueda García ${ }^{5}$, com um intervalo de confiança de 95\% e com erro máximo admissível de 7,5\%. Para alcançar essa amostra, foram entrevistados 67 pacientes no período de setembro de 2006 a junho de 2007.

Um questionário semi-estruturado previamente testado em um estudo piloto foi aplicado aos pacientes no momento da retirada dos medicamentos. Essas entrevistas foram realizadas em sala reservada no próprio serviço de DST/AIDS do município, após a assinatura do termo de consentimento livre e esclarecido.

Os critérios de inclusão foram: ser paciente de qualquer sexo e estar em tratamento com anti-retrovirais. Os critérios de exclusão foram: ter idade menor que 18 anos ou não aceitar participar da pesquisa.

Foram levantadas características como sexo, idade e escolaridade, bem como relativas ao diagnóstico e ao tratamento, tais como nome do medicamento, indicação, dose, frequiência de uso, duração do tratamento, horário de administração, efeitos adversos e precauções. Durante a entrevista, o paciente podia consultar a receita ou qualquer outro material para responder às questões relativas ao medicamento. As datas de retirada, o tempo de diagnóstico e a indicação de tratamento foram coletados no prontuário do paciente.

0 nível de adesão foi determinado mediante: 0 questionamento sobre a administração dos medicamentos anti-retrovirais em determinado período (dia anterior, três dias, sete dias e quinze dias); a verificação das datas de retiradas desses medicamentos (três e seis meses); e a percepção de adesão do paciente. Foram considerados aderentes aqueles pacientes que, de acordo com o método investigado, utilizaram mais de $90 \%$ das doses, que não apresentaram atraso de mais de $10 \%$ do período certo para a retirada da medicação e que se consideraram aderentes segundo a auto-avaliação.

A entrada e a análise desses dados foram feitas nos programas Epi Data 3.0 e Epi Info 6.0. As análises de associação foram realizadas através do teste do qui-quadrado, com nível de significância de 5\%, entre as medidas de adesão e as variáveis sexo, idade, escolaridade, cor da pele, situação de emprego, renda, tempo de infecção, modo de infecção, tempo de uso, esquema de tratamento, estado marital, presença de doenças oportunistas, número de tratamentos já realizados e tempo de tratamento atual.

0 presente trabalho foi aprovado pelo Comitê de Ética da Universidade do Sul de Santa Catarina (UNISUL) sob o registro 06.122.4.03.III.

\section{RESULTADOS}

Características sociodemográficas e culturais. Os entrevistados tinham entre 24 e 65 anos de idade, média de 41,03 anos e desvio padrão (DP) de 9,08; 52,2\% eram homens; 46,3\%, mulheres; e 1,5\%, transexual. Das 31 mulheres, uma era gestante e, no momento da entrevista, estava no oitavo mês de gestação.

Quanto ao comportamento sexual, os mais referidos foram o heterossexual, com $76,1 \%$, e o homossexual, com $14,9 \%$ de frequiência. Quando indagados sobre a cor da pele, 70,1\% referiram ser de etnia branca, e 19,4\%, de parda ou morena. 
A maioria $(65,7 \%)$ dos entrevistados reside na cidade-sede do serviço e possuem casa própria $(73,1 \%)$. A maioria $(73,1 \%)$ reside com a família, dos quais $46,3 \%$ são casados ou têm companheiro; $55,2 \%$ dos pacientes possuem dependentes, uma média de $1,94(\mathrm{DP}=1,13)$. Dos entrevistados, a maioria $(88,1 \%)$ relatou que as famílias tinham conhecimento da doença, e desses $81,4 \%$ recebiam apoio da família.

Dos entrevistados, 46,3\% têm de 4 a 7 anos de escolaridade. Quanto à situação profissional, 25,4\% são assalariados, e 38,8\% são aposentados ou recebem pensão. A renda familiar referida de 64,2\% é de 1 a 2,9 salários mínimos - o valor do salário no período era de 350 reais, aproximadamente 140 dólares.

Em relação ao uso de drogas (lícitas ou ilícitas), 58,2\% relataram usar ou já ter usado algum tipo de droga. Desses pacientes, 33,3\% fazem uso regular (mais de uma vez na semana) de cigarro, 10,3\% de maconha e 7,7\% de álcool. Dos que relataram fazer uso esporádico de drogas (menos de uma vez na semana), 7,7\% fazem uso de maconha e 23,1\% de álcool. Alguns pacientes relataram não tomar a medicação em alguns fins de semana para poder ingerir álcool.

Quando avaliados quanto se a acuidade visual poderia influir na adesão ao tratamento e no acompanhamento de orientações, $55,2 \%$ referiram não possuir qualquer problema.

História médica e situação clínica atual. A média do tempo do diagnóstico dos entrevistados é de 5,8 anos ( $\mathrm{DP}=3,65)$. Quando questionados sobre o tipo de exposição, 74,6\% dos pacientes dizem ter se exposto à doença por meio de relações sexuais. Ao investigar o motivo pelo qual os pacientes descobriram o seu diagnóstico, verificou-se que 53,7\% foram em consequiência do aparecimento de uma doença oportunista.

A maioria (74,6\%) dos pacientes avalia a sua saúde como sendo boa ou excelente, $73,1 \%$ relatam não ter nenhum outro problema de saúde no momento da entrevista. Dos pacientes estudados, 26,9\% referiram possuir outros problemas de saúde, sendo geralmente representados por hipertensão, hepatite $\mathrm{C}$, bronquite, diabetes ou depressão. Além da medicação anti-retroviral, 47,8\% dos entrevistados referiram utilizar outros medicamentos.

A média dos valores de CD4 pré-tratamento foi de 311,64 céls/mm $\mathrm{mm}^{3}(\mathrm{DP}=248,38)$, sendo o atual de 452,71 céls $/ \mathrm{mm}^{3}$ $(\mathrm{DP}=215,93)$. A média da carga viral pré-tratamento foi de $159.586,59$ cópias/mL $(\mathrm{DP}=230.564,47)$, e a atual é de 27.794,28 cópias/mL $(\mathrm{DP}=128.594,28)$, neste caso apresentando mediana de 0,00 . Dos entrevistados que responderam à questão sobre internação hospitalar, 83,8\% relatam não ter realizado nos seis meses anteriores à entrevista.

Terapia farmacológica. 0 principal motivo para o início da terapia anti-retroviral foi por indicação terapêutica (presença de doenças oportunistas em $56,7 \%$ ), seguido por indicação imunológica (diminuição do CD4 sem presença de doença oportunista em $25,4 \%$ ).

Dos pacientes entrevistados, 47,8\% já mudaram de tratamento pelo menos uma vez. As causas mais frequientes para a mudança foram as reações adversas $(32,4 \%)$ e a falência clínica $(12,7 \%)$.
Geralmente, a terapia anti-retroviral (TARV) é composta de dois ou mais medicamentos. No grupo em estudo, $62,7 \%$ utilizam dois medicamentos, ressalta-se que a maioria dos pacientes utilizava medicamentos composto pela associação de dois princípios ativos em uma única forma farmacêutica, sendo neste caso contato como um único medicamento. Quanto à avaliação do número de medicamentos, $67,2 \%$ avaliaram o número de medicamentos como excelente. Ainda, $72,7 \%$ avaliam o tratamento como adequado ao seu estilo de vida, e 85,1\% avaliam o horário de administração dos medicamentos como excelente.

Os pacientes estão no tratamento atual há pelo menos quinze dias e por até nove anos, sendo a média de tempo do tratamento atual de 28,56 meses $(\mathrm{DP}=24,91)$.

As associação de medicamentos mais utilizada por esse grupo de pacientes é efavirenz, zidovudina e lamividuna (26,9\%), atazanavir, zidovudina e lamividuna $(14,9 \%)$, e nelfinavir, zidovudina, lamividuna e nelfinavir (7,5\%). Ressalta-se que a associação de zidovudina com lamivudina é utilizada por $62,8 \%$ dos entrevistados, neste caso fazendo parte do esquema terapêutico junto com outros anti-retrovirais.

0 paciente é o responsável por sua medicação para $91 \%$ dos entrevistados; para os outros, o parceiro ou a mãe é o responsável.

Uma parcela $(49,3 \%)$ dos entrevistados disse não ter dificuldades para ingestão dos medicamentos. Entre os que relataram dificuldades, as que mais se destacam foram efeitos colaterais (15 casos), esquecimento (10 casos) e cuidados alimentares ( 4 casos).

Um percentual de $84,8 \%$ dos entrevistados avalia a sua relação com a equipe de saúde como excelente.

Adesão à terapia anti-retroviral. Os percentuais encontrados de adesão entre os entrevistados são apresentados na Tabela 1. As variáveis que se mostraram associadas significativamente com a adesão estão apresentadas na Tabela 2 e são representadas pela escolaridade (mais de oito anos de estudo); pelo fato de viver com a família, e não sozinho; por referir uma boa adesão; pela avaliação positiva da TARV; por ter tido o diagnóstico por doença oportunista; pela menor contagem de linfócitos T CD4 (NADIR) ser maior que 200 céls $/ \mathrm{mm}^{3}$; e pelo fato de ser o primeiro tratamento.

Não foi observada associação entre a adesão medida por diversas maneiras e as variáveis relacionadas ao perfil do paciente, como idade, comportamento sexual, sexo, tipo de moradia, cor da pele, renda familiar, problemas de visão, possuir dependentes, estado civil e uso de drogas; com o perfil clínico, como presença de doenças oportunistas no momento, com o $\mathrm{CD} 4$ pré-tratamento, com a carga viral atual, com a forma de exposição ao vírus, com o fato de ter sido hospitalizado nos últimos seis meses, com o índice de massa corpórea e com o tempo de diagnóstico; com $o$ tratamento anti-retroviral, como o número de medicamentos em uso, o fato de já ter feito tratamentos anteriores com anti-retroviral, a avaliação do TARV quanto aos horários das medicações, a avaliação do TARV quanto ao estilo de vida, o fato de conhecer a doença (necessidade de tratamento e vias de 


\section{TABELA 1}

Dados relacionados à adesão ao tratamento dos pacientes doentes de AIDS atendidos no Centro de Atendimento Especializado em Saúde.

\begin{tabular}{|c|c|c|}
\hline \multirow[b]{2}{*}{ Forma de avaliação da adesão } & \multicolumn{2}{|c|}{ Aderentes } \\
\hline & $\mathrm{Fi}$ & $\operatorname{Fr}(\%)$ \\
\hline Atraso menor que $10 \%$ na retirada dos medicamentos nos últimos 3 meses $\left(n^{0}=64\right)$ & 36 & 56,3 \\
\hline Atraso menor que $10 \%$ na retirada dos medicamentos nos últimos 6 meses $\left(n^{0}=61\right)$ & 32 & 47,5 \\
\hline Porcentagem de adesão ao tratamento nos últimos 15 dias $\left(n^{0}=46\right)$ & 37 & 80,4 \\
\hline Porcentagem de adesão ao tratamento na última semana $\left(\mathrm{n}^{\circ}=46\right)$ & 37 & 80,4 \\
\hline Porcentagem de adesão ao tratamento nos últimos 3 dias $\left(n^{0}=46\right)$ & 35 & 76,1 \\
\hline Porcentagem de adesão ao tratamento no último dia $\left(\mathrm{n}^{0}=50\right)$ & 35 & 70,0 \\
\hline Como você julga a sua adesão* (de 1 a 5) $\left(n^{\circ}=66\right)$ & 48 & 72,7 \\
\hline
\end{tabular}

*o valor apresentado é dos pacientes que conferiram nota máxima a sua adesão, Fi: frequiência absoluta, Fr: frequiência relativa

TABELA 2

Resultados de associações significativas entre adesão e algumas variáveis dos pacientes doentes de AIDS atendidos no Centro de Atendimento Especializado em Saúde.

\begin{tabular}{|c|c|c|c|c|c|c|c|}
\hline & $\begin{array}{l}\text { Adesão } \\
1 \mathrm{dia}\end{array}$ & $\begin{array}{l}\text { Adesão } \\
3 \text { dias }\end{array}$ & $\begin{array}{l}\text { Adesão } \\
7 \text { dias }\end{array}$ & $\begin{array}{l}\text { Adesão } \\
15 \text { dias }\end{array}$ & $\begin{array}{c}\text { Adesão calculada } \\
\text { pelo atraso por } \\
3 \text { meses }\end{array}$ & $\begin{array}{c}\text { Adesão calculada } \\
\text { pelo atraso } \\
\text { por } 6 \text { meses }\end{array}$ & $\begin{array}{c}\text { Adesão } \\
\text { auto-referida }\end{array}$ \\
\hline & \multicolumn{7}{|l|}{ Valor de p } \\
\hline Ter estudado mais de 8 anos & 0,2798 & 0,3556 & 0,6911 & 0,6038 & 0,0517 & 0,0026 & 0,7219 \\
\hline Viver com a família & 0,9439 & 0,6094 & 0,4600 & 0,0248 & 0,7996 & 0,6862 & 0,0216 \\
\hline Avaliação excelente do tratamento atual & 0,0543 & 0,0935 & 0,7063 & 0,2292 & 0,2404 & 0,2844 & 0,0190 \\
\hline Referir a adesão como excelente & 0,0039 & 0,5483 & 0,0057 & 0,0000 & 0,3255 & 0,2353 & - \\
\hline Ter o diagnóstico do HIV devido a uma doença oportunista & 0,2758 & 0,0935 & 0,0107 & 0,0160 & 0,0365 & 0,0208 & 0,8135 \\
\hline Ter NADIR acima de 200 células por $\mathrm{mm}^{3}$ & 0,8446 & 0,9299 & 0,2594 & 0,0269 & 0,8789 & 0,7955 & 0,0047 \\
\hline Estar realizando o primeiro tratamento & 0,0189 & 0,0387 & 0,2071 & 0,4151 & 0,4985 & 0,6112 & 0,0818 \\
\hline
\end{tabular}

HIV: vírus da imunodeficiência adquirida, NADIR: menor contagem de linfócitos CD4 do paciente.

transmissão), o conhecimento da importância da adesão, a pessoa que responde pelo tratamento (paciente ou cuidador), e reações adversas pregressas e atuais; e outros, como a avaliação da equipe de saúde do serviço e o fato de os familiares conhecerem o diagnóstico.

\section{DISCUSSÃo}

Diferentes métodos foram utilizados para avaliar a adesão, sendo observadas diferenças entre o descrito pelo paciente e 0 calculado pela data de retirada, tendo sido os últimos valores de adesão os menores. Cerca de $70 \%$ a $80 \%$ dos pacientes apresentam adesão, considerando-se aderentes os pacientes que ingeriram pelo menos $90 \%$ das doses prescritas. Um valor semelhante foi encontrado na adesão referida pelo paciente. Contudo, a adesão segundo as datas de retirada dos medicamentos foi em torno de $50 \%$.

Atualmente, não há um padrão-ouro para medir aderência. A dosagem da concentração sérica dos medicamentos pode ser afetada pelo tempo de meia-vida da medicação. Além do custo elevado, não se oferece informação acurada sobre a última dose tomada pelo paciente. A monitoração eletrônica dos frascos também possui um custo elevado e tende a superestimar a aderência, pois os pacientes podem abrir os frascos e não tomar a medicaçã $0^{15}$.
Entrevistas e verificação das datas de retirada também tendem a superestimar a aderência, uma vez que dificilmente o paciente assume para o profissional de saúde que não está tomando as medicações por medo de represália, mas suas vantagens são o baixo custo e a facilidade de obter-se a informaçã $0^{15}$.

É bastante complexo avaliar a aderência a medicamentos, pois nenhum dos métodos disponíveis é totalmente satisfatório. 0 auto-relato tem sido bastante utilizado, mas o ideal parece-nos ser a associação de mais de um método. Na verificação da adesão por meio do auto-relato há a presença do viés da recordação.

Por causa disso e dos outros fatores citados anteriormente, os valores de adesão encontrados neste estudo, realizados através do autorelato, poderiam estar superestimados, o que reforçou a necessidade de cruzamento desse tipo de dados com a verificação das datas de retirada. Depois do cruzamento, as datas de retirada confirmaram a superestimativa da adesão obtida com o auto-relato, uma vez que os valores de adesão determinados com o primeiro método foram de $20 \%$ a 30\% menores que os registrados pelo segundo.

Por meio do auto-relato, os índices, para ser considerados aderentes, variam de $80 \%$ a $95 \%$ da ingesta das doses diárias em determinado tempo, que pode ser de dois ${ }^{17}$, três ${ }^{115}$, sete ${ }^{13} \mathrm{e}$ trinta dias $^{10}$ ou até durante todo o tratamento ${ }^{3}$. Para um índice de $80 \%$ de adesão, a taxa de aderentes encontrados é de $69 \%{ }^{15}$, ou de $75,8 \%{ }^{13}$, ou de $83,2 \%^{3}$. Para um índice de $90 \%$, o percentual de aderentes foi de $74 \%{ }^{10}$. Para um índice de $95 \%$ de adesão, a taxa de aderentes encontrados é de $49 \%{ }^{17}$, ou de $71 \%^{1}$, ou de $76 \%{ }^{3}$. 
Neste estudo, as variáveis que se mostraram associadas com a adesão e relacionadas ao perfil do paciente foram a escolaridade (mais de oito anos de estudo) e o fato de viver com a família, e não sozinho.

Em outros estudos, a escolaridade também foi associada a uma melhor adesão ao tratamento ${ }^{31012}{ }^{15}$. Tal fato pode ter relação com a dificuldade de entendimento dos pacientes com nível cultural mais baixo, principalmente em se tratando de tratamento tão complexo.

Muitos pacientes têm resistência em contar o diagnóstico da doença aos seus familiares, o que compromete o apoio familiar, que poderia influenciar positivamente no sucesso da terapia. Inclusive, apenas o fato de viver com a família parece tornar os pacientes mais empenhados em seguir o tratamento. Por exemplo, no estudo de Carvalho ${ }^{3}$, a estrutura familiar e/ou comunitária também foi associada a uma maior adesão.

Outras variáveis significativamente associadas à adesão e relacionadas ao perfil do paciente encontradas em outros estudos foram: idade ${ }^{1310}$, situação de emprego, renda pessoal e/ou familiar e uso de substâncias ilícitas ${ }^{3}$.

A associação da adesão com a história clínica do paciente neste estudo foi significativa com as variáveis: por ter tido o diagnóstico por doença oportunista; pelo fato de ser o primeiro tratamento; pelo NADIR ser maior que 200 céls $/ \mathrm{mm}^{3}$; por referir uma boa adesão; e pela avaliação positiva da terapia anti-retroviral.

A presença de infecção oportunista no momento do diagnóstico parece sensibilizar o paciente para o tratamento, uma vez que ele pode perceber que sem o tratamento outras doenças oportunistas podem aparecer. Tal variável também foi associada com uma maior adesã $0^{3}$.

O tempo de tratamento não é uma variável consensual na literatura em relação a uma maior predição de adesão. Havia pacientes que se tratavam há menos de oito meses e tiveram cerca de três vezes mais chances de não aderir quando comparado a pacientes que se tratavam há mais de oito meses. Isto pode ser justificado pelo fato de os pacientes tornarem-se mais empenhados em seguir o tratamento a partir do momento que percebem ganhos na sua condição clínica, em função dos medicamentos ${ }^{10}$. No entanto, também se encontrou piora na adesão à medida que 0 tempo de tratamento aumentou ${ }^{22}$.

Neste estudo, não foi verificada a associação da adesão com o tempo, e sim com o primeiro tratamento. Tal associação pode estar relacionada a uma maior expectativa do paciente com 0 sucesso da terapia no início do tratamento, o que pode diminuir com o tempo ou com a não-avaliação de melhora da sua qualidade de vida ou do seu quadro clínico.

A avaliação positiva da terapia anti-retroviral aumenta as chances de adesão. Diferentes estudos têm demonstrado como motivo da não-adesão fatores relacionados ao número de medicamentos ou doses diárias ${ }^{2}{ }^{12}{ }^{17}$, restrição dietética ${ }^{2}$ e efeitos adversos ${ }^{381213}$.

Fatores ligados à medicação podem ser superados, por exemplo, adaptando-se os horários do tratamento ou com a troca de medicação ${ }^{12}$. Além disso, alguns avanços tecnológicos reduziram dificuldades referentes ao uso da medicação, como o número de comprimidos diários, a exemplo da associação de lamivudina e zidovudina. Contudo, é importante estimular o paciente a adaptar a sua rotina diária ao regime terapêutico, reforçando-se a importância da aderência a cada encontro.

As dificuldades relacionadas aos efeitos colaterais, apesar de importantes ${ }^{18}$, são insuficientes para explicar a não-adesão ao tratamento ${ }^{12}$. Por outro lado, parece mais difícil encontrar soluções técnicas para as relacionadas aos fatores sociais, como o estigma, uma vez que fazem parte de uma dinâmica social mais ampla. Dessa maneira, além de assegurar o acesso ao tratamento, é necessário melhorar a qualidade do cuidado e também investir nas políticas sociais e culturais que afetam os pacientes que vivem com o HIV/AIDS.

Acredita-se que a aceitação da adesão à terapia anti-retroviral também pode ser influenciada pela confiança depositada no sistema de saúde e na relação interpessoal com os profissionais de saúde. Desse modo, o papel do profissional que presta assistência é importante, principalmente pela capacidade de diálogo e de negociação. Orientar sobre a doença, a importância da adesão, os medicamentos, o modo correto de usá-los, os efeitos colaterais do tratamento e as medidas a serem tomadas quando tais efeitos ocorrem constitui um aspecto prioritário no tratamento e deve envolver todos os profissionais de saúde responsáveis pela assistência ${ }^{12}{ }^{18}$.

Como já mencionado, para auxiliar o paciente a melhorar a sua adesão ao tratamento, muitas estratégias têm sido descritas na literatura. Algumas delas devem ser implementadas com rigor, como a formação de grupos de adesão mais efetivos ou grupos de assistência individual que respondam às expectativas e às dúvidas decorrentes do uso dos anti-retrovirais, o fornecimento gratuito de lembretes, de tabelas de horários, assim como diários de uso dos medicamentos e pills-boxes.

Um estudo propôs a adoção de orientações individuais com 0 auxílio de um roteiro ilustrado para a realização das ações relativas aos medicamentos ${ }^{8}$. Em outro estudo, foi adotado um programa de assistência terapêutica domiciliar e verificou-se que os pacientes desse programa apresentaram significativamente maior adesão quando comparado ao tratamento ambulatorial convencional ${ }^{9}$.

0 que se verifica na prática é que diferentes estratégias podem ser utilizadas para promover a adesão, contudo o importante é que o desenvolvimento e a implementação dessas intervenções sejam realisticamente desenhados para grupos específicos, levando em consideração as características individuais, o estilo de vida e o suporte social ${ }^{8}$.

Considera-se como limitação deste trabalho o fato de terem sido apenas entrevistados pacientes que procuraram o serviço onde foi aplicado o questionário, o que pode ter aumentado a porcentagem de adesão encontrada, pela possibilidade de os pacientes abordados serem os que utilizam a medicação e, portanto, procuram o serviço para acompanhar a sua doença e retirar a medicação.

Não foram feitas considerações acerca do respeito ao horário de ingesta da medicação ou do seguimento das eventuais restrições dietéticas necessárias, o que deve ser levado em consideração 
em estudos que avaliam a adesão, pois existe dificuldade de manutenção dessas rotinas por longos períodos, o que também pode influenciar no sucesso do tratamento.

É importante salientar que ao final desta pesquisa os resultados deste trabalho foram apresentados a equipe de saúde e buscasse no momento a construção de estratégias para a melhoria dos resultados encontrados.

\section{AGRADECIMENTOS}

Os autores agradecem a colaboração dos outros profissionais que atuam no Centro de Especialidades em Saúde, em especial aos pacientes sujeitos desta pesquisa.

\section{REFERÊNCIAS}

1. Barroso LLMMB. Adesão ao tratamento com anti-retrovirais entre pacientes com aids. Online Brazilian Journal of Nursing 5:22-26, 2006.

2. Brito AM, Szwarcwald CL, Castilho EA. Fatores associados à interrupção de tratamento anti-retroviral em adultos com Aids, Rio Grande do Norte, Brasil, 1999-2002. Revista da Associação Médica Brasileira 52:86-92, 2006.

3. Carvalho CV, Duarte DB, Merchán-Hamann E, Bicudo E, Laguardia J. Determinantes da aderência á terapia anti-retroviral combinada em Brasília, Distrito Federal, Brasil, 1999-2000. Caderno de Saúde Pública 19:593-604, 2003.

4. Carvalho CV, Merchán-Hamann E, Matsushita R. Determinantes da adesão ao tratamento anti-retroviral em Brasília, DF: um estudo de caso-controle. Revista da Sociedade Brasileira de Medicina Tropical 40:555-565, 2007.

5. Cebrián AA, Garcia MMR. Tamaño y selección de muestras en poblaciones finitas. Pharmaceutical Care España 2:310-320, 2000

6. Chesney MA. Factors affecting adherence to antiretroviral therapy. Clinical Infections Diseases 30:171-176, 2000.

7. Desai N, Mathur M. Selective transmission of multidrug resistant HIV to a newborn related to poor maternal adherence. Sexual Transmition Infections 79:419-421, 2003.

8. Figueiredo RM, Sinkoc VM, Tomazim CC, Gallani MCBJ, Colombrini MRC. Adesão de pacientes com AIDS ao tratamento com anti-retrovirais: dificuldades relatadas e proposições de medidas atenuantes em um Hospital Escola. Revista Latino Americana de Enfermagem 9:50-55, 2001.
9. Gupta N, Silva ACS, Passos LN. The role of integrated home-based care in patient adherence to antiretroviral therapy. Revista da Sociedade Brasileira de Medicina Tropical 38:241-245, 2005.

10. Lignani Júnior L, Greco DB, Carneiro M. Avaliação da aderência aos antiretrovirais em pacientes com infecção pelo HIV/AIDS. Revista de Saúde Pública 35:495-501, 2001.

11. Lowe SH, Prins JM, Lange JMA. Antiretroviral therapy in previously untreated adults infected with the human immunodeficiency virus type I: established and potential determinants of virological outcome. The Netherlands Journal of Medicine 62:424-440, 2004.

12. Melchior R, Nemes MIB, Alencar TMD, Buchalla CL. Desafios da adesão ao tratamento de pessoas vivendo com HIV/Aids no Brasil. Revista de Saúde Pública 41:87-93, 2007.

13. Monreal MTFD, Cunha RV, Trinca LA. Compliance to antiretroviral medication as reported by AIDS patients assisted at the University Hospital of the Federal University of Mato Grosso do Sul, The Brazilian Journal of infectious Diseases 6:8-14, 2002.

14. Montessori V, Press N, Harris M, Akagi L, Montaner JSG. Adverse effects of antiretroviral therapy for HIV infection. Canadian Medical Association Journal 170:229-238, 2004

15. Nemes MIB, Souza MFM, Kalichman AO, Grangeiro A, Souza RA, Lopes JF. Avaliação da aderência ao tratamento por anti-retrovirais em usuários de ambulatórios do sistema público de assistência à Aids no Estado de São Paulo. Ministério da Saúde, Brasília, 2000.

16. Remor E, Milner-Moscovics J, Preussler G. Adaptação Brasileira do "Questionario para la evaluación de la adhesión al tratamiento anti-retroviral. Revista de Saúde Pública 41:685-694, 2007.

17. Silveira VL, Drachler ML, Leite JCC, Pinheiro, CAT. Characteristics of HIV Antiretroviral Regimen and Treatment Adherence. Brazilian Journal Infectious Disease 7:194-201, 2003.

18. Souza FG, Mello RS, Galato D. Percepção de pacientes com HIV sobre o uso de Enfuvirtida: estudos de casos realizados em um Centro de Saúde de Santa Catarina. Revista Brasileira de Farmácia 84: 308-310, 2008

19. United Nations on AIDS. Boletim epidemiológico, Ministério da Saúde, 2007. Disponível em: http://www.onu-brasil.org.br/doc/BoletimEpidemigico2007Release.doc Acesso em: 2 jun 2008.

20. Vitória MAA. Conceitos e recomendações básicas para melhorar a adesão ao tratamento anti-retroviral. Ministério da Saúde. p. 1-5, 1998. Disponível em: http://bvsms.saude.gov.br/bvs/publicacoes/15conceitos_rec_basicas.pdf Acesso em: 8 abr. 2008

21. World Health Organization, Adherence to long-term therapies: evidence for action, Switzerland: Publications, 2003

22. Willians AB. Adherence to highly active antiretroviral therapy. The Nursing Clinics of North American 34:113-129, 1999. 